\title{
PSYCHOLOGICAL IMPACT OF COVID-19 PANDEMIC ON HEALTH CARE WORKERS
}

\author{
By \\ Hathout HM${ }^{1}$, El-Esrigy FA² ${ }^{2}$ Farag $\mathrm{NA}^{2}$ and El Dalatony $\mathrm{MM}^{1}$ \\ ${ }^{1}$ Department of Public Health and Community Medicine, ${ }^{2}$ Department of Family Medicine ,Faculty of \\ Medicine, Menoufia University, Menoufia, Egypt. \\ Corresponding author: Hathout HM.Email: hanan.hathout@med.menofia.edu.eg \\ Submit Date: 2020-12-05 \\ Revise Date: 2020-12-14 \\ Accept Date: 2020-12-17
}

DOI: 10.21608 / ejom. 2020.52274 .1218

Authors' contribution: Hathout HM and Elsrigy FA shared in the design of the study, in analysis and interpretation of data. Farag NA shared in providing research materials. El Dalatony MM shared in editing. All authors shared in drafting the article and have critically reviewed and approved the final draft.

\begin{abstract}
Introduction: Healthcare staffs are first-line fighters who handle COVID-19 patients. They face a high risk of infection every day, and are subject to long and distressing work hours in order to meet health needs. Aim of work: To evaluate the psychological impact of COVID-19 pandemic on health care workers (HCWs). Materials and Methods: All categories of health care workers in Egypt were invited to participate in the study through sharing the link to online professional groups between 21st June to 9th July 2020. The survey included a self-administered- Arabic questionnaire including information related to socio- demographic characteristics, medical history including diseases and medications, work characteristics and COVID -19 experience of the studied participants. The survey also included validated Depression, Anxiety, and Stress Scales (DASS-21) and 7 items work-related burnout Copenhagen Burnout Inventory (CBI). Results: The total number of health care workers who shared in the study was 764 . There were psychological disorders of varying degrees-in the form of depression (41.5\%), anxiety (67\%), and stress (27.7\%). Working as nurses, age < 40 years, changing vacation plans during COVID 19, shift work, less than 10 years of experience, single HCWs were the most significant factors for predicting stress. Conclusion: The results concluded that health care workers had varying degrees of psychological stress related to the job, particularly those in direct relationships with
\end{abstract}


COVID-19 patients and those who work in fever and chest hospitals. Psychological support for promoting mental well-being in health care workers exposed to COVID-19 should be implemented immediately, particularly for female, nurses and frontline health care workers.

Key words: Psychological impact, Depression, Anxiety, and Stress Scales (DASS), Burnout, COVID-19 and Health Care Workers (HCWs).manifestations.

\section{Introduction}

Pandemics and epidemics are an emergency in public health and can lead to severe deaths and socio-economic disruption (Fernandez et al., 2020). Several outbreaks of new infectious diseases have occurred worldwide in the last 20 years, such as the outbreak of Severe Acute Respiratory Syndrome (SARS) in 2002 and the influenza pandemic H1N1 in 2009-2010. Pandemics require intense and rapid health-care response, with thousands of health-care workers (HCWs) working either directly or indirectly with patients, fighting at the frontline to address the challenges posed to healthcare systems (Preti et al., 2020).

Nowadays the world is suffering from severe acute respiratory syndrome Coronavirus-2 (SARS-CoV-2), the causative agent of (COVID-19) first reported in China on December 31, 2019. The World Health Organization (WHO) announced the condition as a worldwide pandemic on March 11, 2020. Health Care Workers (HCWs) are experiencing physical and psychological fatigue under the enormous stress of this pandemic (Temsah et al., 2020). Medical staff often has a variety of psychological problems under high pressure and risk (Kang et al., 2015). In pandemic situations, work overload, physical exhaustion, shortage of Personal Protective Equipment (PPE), nosocomial transmission, sometimes, in addition to the difficult decisions needed to save lives, have resulted in dramatic physical and mental effects on HCWs. The latter are therefore at higher risk of developing mental health problems, such as fear, anxiety, depression, and insomnia (Papa et al., 2020). In China, frontline healthcare workers exhibited high prevalence of severe symptoms of mental health (Blake et al., 2020).

\section{Aim of work}

Evaluation of COVID-19 pandemic's psychological impact on health care workers.

\section{Materials and Methods}

-Study Design: This is a cross- 
sectional study targeting health care workers through distributing of an online survey.

-Place and duration of the study: This work was conducted in Egypt during the period between 21 June to 9 July 2020 during COVID 19 pandemic period following the declaration of the World Health Organization (WHO) that the disease is a Public Health Emergency of International Concern on January 30, 2020 (Barry et al., 2020).

Study Sample: The sample size was calculated at $95 \%$ confidence interval using the (Open-Epi Version 3, open source calculator-SSPropor) software developed by Centers for Disease Control and Prevention (CDC, 2013). The number of health care workers in Egypt according to the WHO (2018) is 264.249 including 77.600 medical doctors, 135.88 nurses, 29.100 pharmacists, 19.400 dentists, and 2349 technicians (WHO, 2018).

The minimum required sample size was calculated as 383 participants. But, due to availability of the survey online on professional medical groups and wide spread use of social media; the number of participants was exceeding the recommended sample size. The total number of HCWs participated were 764 .
Pilot study: Included a convenient sample of twenty accepted subjects to measure feasibility of study setting, content, and time for filling of the tools then excluded from the study. The average time for filling the survey is 7 minutes.

Inclusion criteria: Health care workers (HCWs) of all specialties including dentistry, laboratory, pharmacy, nursing, etc. working in different hospitals (Ministry of Health, University, Teaching...etc.) and health care centers either governmental or private. Also, health care workers of all clinical departments including the outpatient clinic, emergency, inpatient departments, critical care units, laboratory, and pharmacy (whether involved or not in the management of suspected/ confirmed COVID-19 cases).

-Study method: The study team shared the online survey using google forms through various social network groups of different HCW's professional specialties on Facebook, WhatsApp, Instagram as medical syndicate's pages on Facebook, Family Medicine Union in Egyptian Universities, Union of medical professionals, Egypt nurses, Menoufia Medical School graduates, group of white coats, etc. 
The team explained the purpose of the study to all participants prior to conducting the survey, participants registered through the link using their personal emails. Therefore, no participant could participate more than one time.

Questionnaires: The survey included a self-administered- Arabic questionnaire including sociodemographic data, medical history including diseases and medications, questions regarding work characteristics and COVID -19 experience of the studied participants. The survey also included the validated Depression, Anxiety, and Stress Scales (DASS-21) and seven items work-related burnout Copenhagen Burnout Inventory (CBI).

-The Depression, Anxiety and Stress (DASS) questionnaire: 21 items scale has three subscales assessing anxiety ( 7 items), depression ( 7 items) and stress ( 7 items). The Arabic version of the questionnaire is used to assess participant's response to various domains (Lovibond and Lovibond 1995). We asked the participants to read each statement and circle a number $0,1,2$ or 3 , which indicates how much the statement applied to them over the past week. The rating scale is as follows: zero (NEVER), one (SOMETIMES), two (OFTEN), three (ALMOST ALWAYS). We calculated scores for depression, anxiety, and stress by summing the scores for the relevant items. We multiplied Scores on the DASS- 21 by 2 to calculate the final score. Then we categorized the final scores for each domain (Depression, Anxiety, and stress) as follows:

- For depression: Normal range (0-9), Mild Depression (10-13), Moderate Depression (14-20), severe Depression (21-27), and Extremely Severe Depression is equal or more than 28 .

- For Anxiety: Normal range (0-7), Mild Anxiety (8-9), Moderate Anxiety (10-14), Severe Anxiety (1519), and Extremely Severe Anxiety is equal or more than 20 .

- For Stress: Normal range (014), Mild Stress (15- 18), Moderate Stress 19-25), Severe Stress (26-33), and Extremely Severe Stress is equal or more than 33 .

- Seven items work-related burnout Copenhagen Burnout Inventory (CBI): The work-related burnout questionnaire composed of seven items. The answers to each item 
taking a 5 items Likert scale ranging from "always or to a very high degree, often or to a high degree, "sometimes or somewhat, seldom or to a low degree, and never/almost never or to a very low degree". High CBI score denotes higher degree of stress and burnout (Sestili et al., 2018)

\section{Consent}

An informed consent to participate in the study was included at the beginning of the online survey. Confidentiality of the data was ensured through questionnaire anonymity.

\section{Ethical approval}

The Institutional Ethical Committee Board of the Faculty of Medicine, Menoufia University, Egypt approved the study. Questionnaire anonymity ensured the confidentiality of data (IRB No: 7RB 8/2020 FAML).

\section{Data management}

Data were analyzed using Statistical Package of Social Science (SPSS) version 23 (using IBM personal computer). Quantitative data were expressed as mean and standard deviations $(\mathrm{X} \pm \mathrm{SD})$ and analyzed by student $\mathrm{t}$-test-or kruskall wallis test $(\mathrm{k})$. We expressed and analyzed qualitative data as number and percentage using Chi-square test. Multi-variate regression analysis model was done to test the ability of different variables (e.g. age, sex, marital status, history of chronic diseases and history of antiepileptic medications), etc. to predict psychological disturbance in the studied HCWs. 


\section{Results}

The total number of the participants were 746 , most of them were in the age categories $\leq 40$ years $(85 \%)$, female (81\%), and married (79.6\%). No smoking history in $77.7 \%$, and no history of chronic diseases in $72.6 \%$. Most of the participants were medical doctors and dentists $(67.1 \%)$.

Figure (1): Distribution of study participants according to the severity of DASS Subscale.

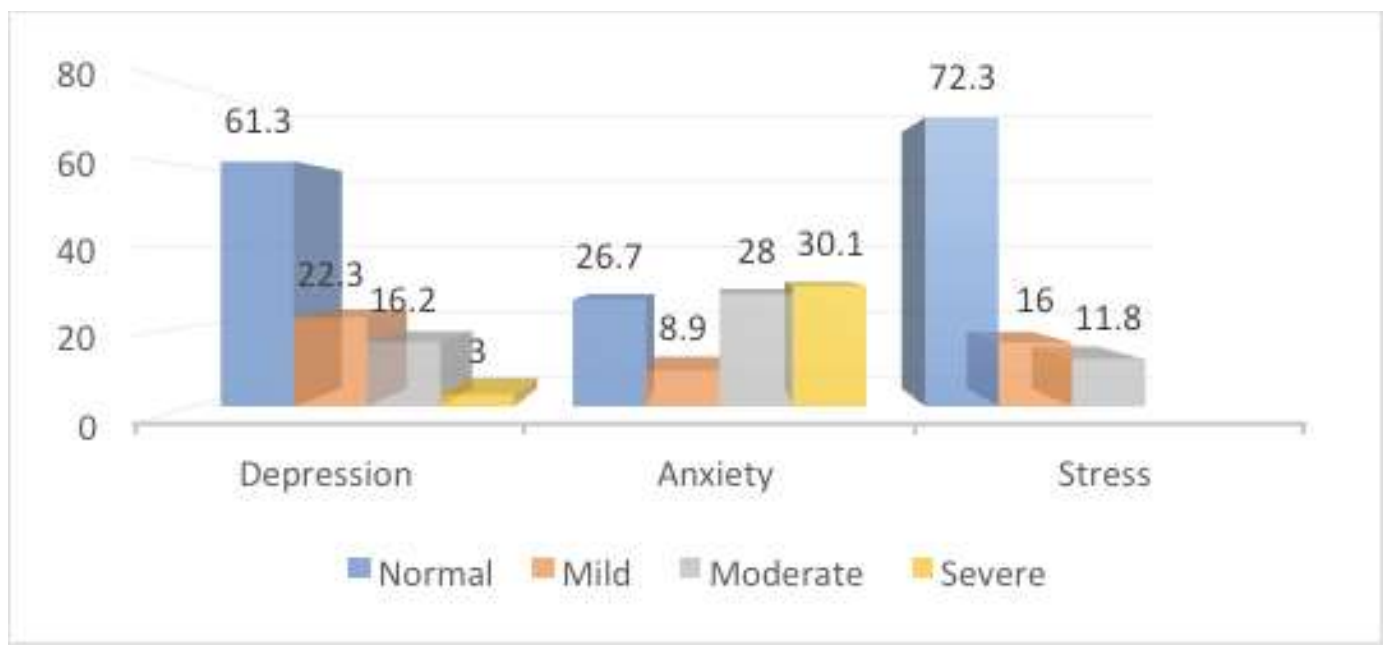

Hundred and seventy participants $(22.3 \%)$ of HCWs reported mild depression and $124(16.2 \%)$ showed moderate depression, moderate anxiety was evident in 214 of HCWs (28\%) and severe anxiety in 230 (30, $1 \%)$. However, $122(16 \%)$ had mild stress and $90(11.8 \%)$ had moderate stress (Fig.1) 
Table (1): Relation between the psychological scores of the studied participants, sociodemographic characteristics and medical history.

\begin{tabular}{|c|c|c|c|c|c|}
\hline \multirow{2}{*}{$\begin{array}{l}\text { Socio-demographic } \\
\text { characteristics }\end{array}$} & \multirow[b]{2}{*}{ No $(\%)$} & \multicolumn{3}{|c|}{ DASS } & \multirow[b]{2}{*}{ CBI } \\
\hline & & Depression & Anxiety & Stress & \\
\hline $\begin{array}{l}\text { Age /years } \\
-\leq 40 \\
->40\end{array}$ & $\begin{array}{l}652(85.3 \%) \\
112(14.7 \%)\end{array}$ & $\begin{array}{c}8.7 \pm 4.6 \\
7.04 \pm 3.5\end{array}$ & $\begin{array}{c}12.4 \pm 5.7 \\
9.2 \pm 4.6\end{array}$ & $\begin{array}{c}10.7 \pm 6.1 \\
7.2 \pm 4.6\end{array}$ & $\begin{array}{l}23.1 \pm 4.7 \\
20.9 \pm 5.1\end{array}$ \\
\hline t-test, $p$ value & & $3.7(<0.001)^{*}$ & $5.5(<0.001)^{*}$ & $5.9(<0.001)^{*}$ & $4.5(<0.001)^{*}$ \\
\hline $\begin{array}{l}\text { Gender } \\
\text { - Male } \\
\text { - Female }\end{array}$ & $\begin{array}{l}146(19.1 \%) \\
618(80.9 \%)\end{array}$ & $\begin{array}{l}7.3 \pm 3.9 \\
8.7 \pm 4.5\end{array}$ & $\begin{array}{l}10.1 \pm 5.8 \\
12.3 \pm 5.6\end{array}$ & $\begin{array}{c}8.6 \pm 6 \\
10.6 \pm 5.9\end{array}$ & $\begin{array}{l}21.1 \pm 5.2 \\
23.4 \pm 5.9\end{array}$ \\
\hline t-test, $p$ value & & $10.8(<0.001)^{*}$ & $18.05(<0.001)^{*}$ & $13.2(<0.001)^{*}$ & $18.2(<0.001)^{*}$ \\
\hline $\begin{array}{l}\text { Marital status } \\
\text { - Single / divorced } \\
\text { - Married }\end{array}$ & $\begin{array}{l}156(20.4 \%) \\
608(79.6 \%)\end{array}$ & $\begin{array}{l}9.3 \pm 4.2 \\
8.2 \pm 4.5\end{array}$ & $\begin{array}{l}13.1 \pm 5.4 \\
11.6 \pm 5.7\end{array}$ & $\begin{array}{l}11.5 \pm 5.7 \\
9.9 \pm 6.1\end{array}$ & $\begin{array}{l}23.8 \pm 4.6 \\
22.6 \pm 4.9\end{array}$ \\
\hline t-test, $p$ value & & $2.6(0.009)^{*}$ & $3.003(0.003)^{*}$ & $3.01(<0.001)^{*}$ & $2.8(0.005)^{*}$ \\
\hline $\begin{array}{l}\text { Smoking history } \\
\text { - Yes } \\
\text { - NO }\end{array}$ & $\begin{array}{l}170(22.3 \%) \\
594(77.7 \%)\end{array}$ & $\begin{array}{c}8.4 \pm 4.5 \\
8.5 \pm 4.46\end{array}$ & $\begin{array}{l}12.3 \pm 5.6 \\
11.8 \pm 5.7\end{array}$ & $\begin{array}{c}9.97 \pm 5.9 \\
10.3 \pm 6.08\end{array}$ & $\begin{array}{l}23.3 \pm 8.7 \\
22.9 \pm 4.7\end{array}$ \\
\hline$p$ value & & $0.006(0.937)$ & $0.863(0.353)$ & $0.363(0.547)$ & $0.645(0.422)$ \\
\hline $\begin{array}{l}\text { History of chronic } \\
\text { diseases } \\
\text { - Yes } \\
\text { - NO }\end{array}$ & $\begin{array}{l}210(27.4 \%) \\
554(72.6 \%)\end{array}$ & $\begin{array}{l}9.03 \pm 4.8 \\
8.2 \pm 4.3\end{array}$ & $\begin{array}{l}12.8 \pm 5.8 \\
11.6 \pm 5.6\end{array}$ & $\begin{array}{c}11.1 \pm 9.9 \\
9.9 \pm 5.9\end{array}$ & $\begin{array}{l}22.6 \pm 4.9 \\
22.8 \pm 4.8\end{array}$ \\
\hline t-test, $p$ value & & $2.1(0.034)^{*}$ & $2.7(0.006)^{*}$ & $2.5(0.014)^{*}$ & $0.431(0.667)$ \\
\hline $\begin{array}{l}\text { History of taking ant } \\
\text { psychotic drugs } \\
\text { - Yes } \\
\text { - NO }\end{array}$ & $\begin{array}{l}80(10.4 \%) \\
684(89.6 \%)\end{array}$ & $\begin{array}{l}9.7 \pm 4.7 \\
8.3 \pm 4.4\end{array}$ & $\begin{array}{c}8.3 \pm 4.4 \\
11.7 \pm 5.8\end{array}$ & $\begin{array}{c}12.1 \pm 5.2 \\
10.05 \pm 6.1\end{array}$ & $\begin{array}{l}24.4 \pm 3.7 \\
22.6 \pm 4.9\end{array}$ \\
\hline t-test, p value & & $2.5(0.012)^{*}$ & $3.4(0.001)^{*}$ & $2.8(0.005)^{*}$ & $3.09(0.002)^{*}$ \\
\hline
\end{tabular}

*: Statistically significant

DASS: Depression, Anxiety, and Stress Scales

CBI : Copenhagen Burnout Inventory

The mean DASS-D, DASS-A, DASS-S, and CBI score were statistically significantly higher in HCWs younger than 40 years of age, female gender, single / 
divorced and those with history of anti-psychotic drugs. HCWs with chronic illness showed statistically significantly higher DASS-D, DASS-A and DASS-S score when compared to those with no history of chronic illness.

Table (2): Relation between the studied participants' psychological scores and their work characteristics.

\begin{tabular}{|c|c|c|c|c|c|}
\hline \multirow{2}{*}{ Work characteristics } & \multirow{2}{*}{ No $(\%)$} & \multicolumn{3}{|c|}{ DASS } & \multirow{2}{*}{ CBI } \\
\hline & & Depression & Anxiety & Stress & \\
\hline $\begin{array}{l}\text { Job categories: - } \\
\text { - Physicians / dentists } \\
\text { - Nurses } \\
\text { - Pharmacists/ Technicians }\end{array}$ & \begin{tabular}{|c|}
$512(67.1 \%)$ \\
$32(4.2 \%)$ \\
$220(28.8 \%)$
\end{tabular} & $\begin{array}{l}8.8 \pm 4.3 \\
9.9 \pm 4.6 \\
7.6 \pm 4.6\end{array}$ & $\begin{array}{l}12.2 \pm 5.2 \\
13.6 \pm 6.4 \\
10.9 \pm 6.5\end{array}$ & $\begin{array}{c}10.3 \pm 5.7 \\
13.1 \pm 6.4 \\
9.6 \pm 6.7\end{array}$ & $\begin{array}{c}23.3 \pm 4.7 \\
22.7 \pm 5.05 \\
21.6 \pm 5.04\end{array}$ \\
\hline ANOVA test, $p$ value & & $7.5(<0.001)^{*}$ & $5.9(0.003)^{*}$ & $5.1(0.006)^{*}$ & $9.09(<0.001)^{*}$ \\
\hline $\begin{array}{l}\text { Work place } \\
\text { - Fever hospitals } \\
\text { - Other governmental hospitals } \\
\text { and centers } \\
\text { - Private hospitals }\end{array}$ & $\begin{array}{c}42(5.5 \%) \\
678(88.7 \%) \\
44(5.8 \%) \\
\end{array}$ & $\begin{array}{c}10.8 \pm 4.9 \\
8.4 \pm 4.4 \\
7.6 \pm 4.7\end{array}$ & $\begin{array}{l}12.8 \pm 4.4 \\
11.9 \pm 5.7 \\
11 \pm 5.9\end{array}$ & $\begin{array}{l}10.5 \pm 4.3 \\
10.3 \pm 6.1 \\
8.9 \pm 6.1\end{array}$ & $\begin{array}{l}24.4 \pm 4.6 \\
22.7 \pm 4.8 \\
21.8 \pm 5.1\end{array}$ \\
\hline ANOVA test, $p$ value & & $6.8(0.001)^{*}$ & $1.1(>\cdot, \cdot 0)$ & $1.05(>\cdot, \cdot 0)$ & $3.3(0.039)^{*}$ \\
\hline $\begin{array}{l}\text { Department of work } \\
\text { - Critical } \\
\text { - Non critical } \\
\end{array}$ & $\begin{array}{l}190(24.9 \%) \\
574(75.1 \%) \\
\end{array}$ & $\begin{array}{l}9.2 \pm 4.1 \\
8.2 \pm 4.6 \\
\end{array}$ & $\begin{array}{l}12.9 \pm 4.9 \\
11.6 \pm 5.9 \\
\end{array}$ & $\begin{array}{l}10.9 \pm 5.7 \\
9.98 \pm 6.1\end{array}$ & $\begin{array}{c}23.9 \pm 3.9 \\
22.4 \pm 5.06\end{array}$ \\
\hline t-test, $p$ value & & $2.5(0.012)^{*}$ & $2.6(0.009)^{*}$ & $1.9(0.056)$ & $3.7(<0.001) *$ \\
\hline $\begin{array}{l}\text { Years of experience } \\
-\quad<10 \\
-\geq 10\end{array}$ & $\begin{array}{l}376(49.2 \%) \\
388(50.8 \%) \\
\end{array}$ & $\begin{array}{l}8.9 \pm 4.6 \\
8.1 \pm 4.3 \\
\end{array}$ & $\begin{array}{c}12.9 \pm 5.7 \\
11 \pm 5.5 \\
\end{array}$ & $\begin{array}{c}11.3 \pm 6.1 \\
9.2 \pm 5.8 \\
\end{array}$ & $\begin{array}{l}23.6 \pm 5.1 \\
21.9 \pm 4.4 \\
\end{array}$ \\
\hline t-test, p value & & $2.5(0.012)^{*}$ & $4.6(<0.001)^{*}$ & $4.7(<0.001) *$ & $4.8(<0.001)^{*}$ \\
\hline $\begin{array}{l}\text { Weekly work hours } \\
-\leq 40 \\
->40\end{array}$ & $\begin{array}{l}606(79.3 \%) \\
158(20.7 \%) \\
\end{array}$ & $\begin{array}{l}8.2 \pm 4.5 \\
9.6 \pm 4.3 \\
\end{array}$ & $\begin{array}{l}11.5 \pm 5.8 \\
13.6 \pm 5.1 \\
\end{array}$ & $\begin{array}{l}9.8 \pm 11.7 \\
11.7 \pm 5.7 \\
\end{array}$ & $\begin{array}{l}22.3 \pm 4.8 \\
24.7 \pm 4.7 \\
\end{array}$ \\
\hline t-test, $p$ value & & $3.6(<0.001)^{*}$ & $4.3(<0.001)^{*}$ & $3.5(<0.001)^{*}$ & $5.9(<0.001)^{*}$ \\
\hline $\begin{array}{l}\text { Work shift } \\
\text { - } \quad \text { Fixed } \\
\text { - } \quad \text { Rotating } \\
\end{array}$ & $\begin{array}{l}508(66.5 \%) \\
256(33.5 \%) \\
\end{array}$ & $\begin{array}{l}8.2 \pm 4.5 \\
9.1 \pm 4.4 \\
\end{array}$ & $\begin{array}{l}11.5 \pm 5.8 \\
12.8 \pm 5.3 \\
\end{array}$ & $\begin{array}{c}10.04 \pm 6.3 \\
10.6 \pm 5.5 \\
\end{array}$ & $\begin{array}{l}21.9 \pm 4.9 \\
24.5 \pm 4.1 \\
\end{array}$ \\
\hline t-test, $p$ value & & $2.7(0.008)^{*}$ & $3.05(0.002)^{*}$ & $1.157(0.248)$ & $7.1(<0.001)^{*}$ \\
\hline
\end{tabular}

Critical departments (ER+ ICU+ Radiology+ clinical pathology)

Non critical departments (no direct exposure to suspected COVID-19 cases) * : Statistically significant DASS: Depression, Anxiety, and Stress Scales

CBI : Copenhagen Burnout Inventory 
Table 2 showed that statistically significant higher DASS-D, DASS-A, DASS-S scores was present among nurses, HCWs in fever and chest hospitals, in critical departments, with less than 10 years of work experience, with more than eight daily working hours and changeable work shifts, compared to others. Physicians showed significant high burnout scores compared to other participants of nurses, pharmacists or technicians.

Table (3): Relation between psychological scores and COVID 19 experience of the studied participants.

\begin{tabular}{|c|c|c|c|c|c|}
\hline \multirow{2}{*}{$\begin{array}{l}\text { COVID } 19 \text { experience of } \\
\text { the studied participants }\end{array}$} & \multirow[b]{2}{*}{ No $(\%)$} & \multicolumn{3}{|c|}{ DASS } & \multirow[b]{2}{*}{ CBI } \\
\hline & & Depression & Anxiety & Stress & \\
\hline \multicolumn{6}{|l|}{$\begin{array}{l}\text { Participation of facility in } \\
\text { management of COVID } 19\end{array}$} \\
\hline Yes & $59077.2 \%)$ & $8.9 \pm 4.4$ & $12.3 \pm 5.5$ & $10.6 \pm 5.9$ & $23.4 \pm 5.7$ \\
\hline $\mathrm{NO}$ & $174(22.8 \%)$ & $6.8 \pm 4.2$ & $10.4 \pm 6.1$ & $8.8 \pm 6.4$ & $21.6 \pm 5.9$ \\
\hline t-test, $p$ value & & $30.6(<0.001)^{*}$ & $15.4(<0.001)^{*}$ & $12.6(<0.001)^{*}$ & $12.8(<0.001)^{*}$ \\
\hline \multicolumn{6}{|l|}{$\begin{array}{l}\text { Receiving COVID } 19 \\
\text { training }\end{array}$} \\
\hline Yes & $154(20.2 \%)$ & $7.4 \pm 3.9$ & $10.6 \pm 5.9$ & $8.9 \pm 5.9$ & $21.9 \pm 4.6$ \\
\hline NO & $610(79.8 \%)$ & $8.7 \pm 4.6$ & $12.3 \pm 5.6$ & $10.6 \pm 6.02$ & $23.3 \pm 6.1$ \\
\hline t-test, $p$ value & & $15.6(0.001)^{*}$ & $11.2(0.001)^{*}$ & $9.99(0.002)^{*}$ & $7.1(0.008)^{*}$ \\
\hline $\begin{array}{l}\text { Directly involved in dealing } \\
\text { with COVID } 19 \text { patients }\end{array}$ & & & & & \\
\hline Yes & $496(64.9 \%)$ & $9.0 \pm 4.5$ & $12.5 \pm 5.5$ & $10.6 \pm 5.9$ & $23.1 \pm 4.7$ \\
\hline NO & $268(35.1 \%)$ & $7.5 \pm 4.3$ & $10.9 \pm 5.8$ & $9.5 \pm 6.2$ & $22.2 \pm 4.98$ \\
\hline t-test, $p$ value & & $4.5(<0.001)^{*}$ & $3.7(<0.001)^{*}$ & $2.6(0.01)^{*}$ & $2.5(0.012)^{*}$ \\
\hline Changing vacation plans & & & & & \\
\hline Yes & $618(80.9 \%)$ & $7.8 \pm 4.2$ & $11.2 \pm 5.6$ & $9.5 \pm 5.9$ & $22.2 \pm 4.9$ \\
\hline NO & $146(19.1 \%)$ & $11.3 \pm 4.3$ & $14.9 \pm 4.9$ & $13 \pm 5.6$ & $25.1 \pm 3.9$ \\
\hline t-test, $p$ value & & $8.9(<0.001)^{*}$ & $7.4(<0.001)^{*}$ & $6.4(<0.001)^{*}$ & $6.7(<0.001)^{*}$ \\
\hline $\begin{array}{l}\text { Death of one of colleague } \\
\text { with COVID } 19 \text { infection }\end{array}$ & & & & & \\
\hline Yes & $144(18.8 \%)$ & $10.7 \pm 4.5$ & $13.9 \pm 4.8$ & $12.2 \pm 5.5$ & $24.5 \pm 5.6$ \\
\hline NO & $618(80.9 \%)$ & $7.9 \pm 4.3$ & $11.4 \pm 5.8$ & $9.8 \pm 6.1$ & $22.6 \pm 5.8$ \\
\hline t-test, $p$ value & & $45.7(<0.001)^{*}$ & $22.03(<0.001)^{*}$ & $19.4(<0.001)^{*}$ & $12.2(<0.001)^{*}$ \\
\hline
\end{tabular}


Table 3 showed that DASS-D, DASS-A, DASS-S and CBI scores were statistically significant higher among HCWs in facilities participating in management of COVID 19, not receiving training regarding dealing with COVID 19 cases / or suspected cases, compared to others. Other significant factors included HCWs directly dealing with COVID 19 patients, change of planned vacations, also history of death of one of their colleagues with COVID 19.

\section{Table (4): Logistic regression analysis for prediction of DASS scale parameters} among the studied participants.

\begin{tabular}{|c|c|c|c|c|c|c|}
\hline \multirow{2}{*}{ Variables } & \multicolumn{2}{|c|}{ DASS-Depression } & \multicolumn{2}{|c|}{ DASS- Anxiety } & \multicolumn{2}{|c|}{ DASS- Stress } \\
\hline & OR (95\% CI) & p value & OR $(95 \%$ CI $)$ & p value & OR (95\% CI) & p value \\
\hline Age $\leq 40$ years & $1.96(1.112-3.438)$ & $0.02^{*}$ & $1.85(1.07-3.22)$ & $0.029^{*}$ & $2.95(1.47-5.89)$ & $.002^{*}$ \\
\hline Being female & $1.84(1.123-3.014)$ & $0.015^{*}$ & $2.71(1.65-4.43)$ & $<0.001^{*}$ & $1.24(0.74-2.07)$ & $>0.05$ \\
\hline Being single / divorced & $1.50(.99-2.27)$ & $>0.05$ & $0.97(0.59-1.58)$ & $>0.05$ & $1.54(1.02-2.34)$ & $.041^{*}$ \\
\hline Having history of diseases & $0.717(.48-1.064)$ & $>0.05$ & $0.68(0.43-1.07)$ & $>0.05$ & $0.79(0.52-1.19)$ & $>0.05$ \\
\hline $\begin{array}{l}\text { History of taking anti- } \\
\text { psychotic drugs }\end{array}$ & $1.87(1.04-3.38)$ & $0.036^{*}$ & $3.68(1.54-8.76)$ & $.003^{*}$ & $1.57(0.86-2.89)$ & $>0.05$ \\
\hline $\begin{array}{l}\text { Professional categories: } \\
\text { - Physicians } \\
\text { - Nurses } \\
\text { - Pharmacists/ Technicians }\end{array}$ & $\begin{array}{l}0.92(.62-1.36) \\
2.09(0.85-5.13)\end{array}$ & $\begin{aligned}> & 0.05> \\
& 0.05\end{aligned}$ & $\begin{array}{l}1.81(1.18-2.78) \\
1.44(0.48-4.31)\end{array}$ & $\begin{array}{c}.006^{*} \\
>0.05\end{array}$ & $\begin{array}{l}0.95(0.63-1.44) \\
3.32(1.35-8.12)\end{array}$ & $\begin{array}{l}>0.05 \\
\mathbf{0 . 0 0 9}^{*}\end{array}$ \\
\hline $\begin{array}{l}\text { Place of work } \\
\text { - Fever hospitals } \\
\text { - Other Governmental health } \\
\text { care facilities } \\
\text { - Private hospitals and centers }\end{array}$ & $\begin{array}{l}2.77(1.35-5.69) \\
0.89(0.423-1.89)\end{array}$ & $\begin{array}{c}.005^{*} \\
>0.05\end{array}$ & $\begin{array}{l}3.23(1.07-9.73) \\
0.87(0.39-1.94)\end{array}$ & $\begin{array}{c}\mathbf{0 . 0 3 8} \\
>0.05\end{array}$ & $\begin{array}{l}0.56(0.22-1.39) \\
1.12(0.52-2.41)\end{array}$ & $\begin{array}{c}>0.05> \\
0.05\end{array}$ \\
\hline $\begin{array}{l}\text { Working in critical } \\
\text { departments }\end{array}$ & $1.29(0.87-1.91)$ & $>0.05$ & $1.88(1.14-3.09)$ & $.013^{*}$ & $0.89(0.59-1.37)$ & $>0.05$ \\
\hline Years of experience $<10$ years & $0.99(0.69-1.42)$ & $>0.05$ & $1.31(0.87-1.98)$ & $>0.05$ & $1.55(1.06-2.26)$ & $0.025^{*}$ \\
\hline $\begin{array}{l}\text { Weekly working hours }<40 \\
\text { hours }\end{array}$ & $0.91(0.57-1.45)$ & $>0.05$ & $\begin{array}{c}0.29(0.16- \\
0.567)\end{array}$ & $<0.001^{*}$ & $0.33(0.20-0.54)$ & $0.001^{*}$ \\
\hline Shift work & $1.22(0.82-1.81)$ & $>0.05$ & $1.16(0.72-1.86)$ & $>0.05$ & $2.02(1.29-3.14)$ & $0.002^{*}$ \\
\hline $\begin{array}{l}\text { Participation of facility in } \\
\text { management of COVID } 19\end{array}$ & $1.54(0.92-2.57)$ & $>0.05$ & $1.15(0.67-1.96)$ & $>0.05$ & $0.97(0.57-1.67)$ & $>0.05$ \\
\hline Receiving COVID-19 training & $2.22(1.21-4.08)$ & $.010^{*}$ & $1.51(0.82-2.77)$ & $>0.05$ & $1.18(0.59-2.33)$ & $>0.05$ \\
\hline $\begin{array}{l}\text { Directly involved in dealing } \\
\text { with COVID } 19 \text { patients }\end{array}$ & $0.96(.59-1.57)$ & $>0.05$ & $1.64(1.05-2.59)$ & $0.032^{*}$ & $1.01(0.65-1.54)$ & $>0.05$ \\
\hline Changing vacation plans & $3.57(2.29-5.58)$ & $<0.001^{*}$ & $3.68(1.88-7.19)$ & $<0.001^{*}$ & $2.42(1.55-3.77)$ & $0.000^{*}$ \\
\hline $\begin{array}{l}\text { Death of one of colleague with } \\
\text { COVID } 19 \text { infection }\end{array}$ & $2.01(1.32-3.08)$ & $.001^{*}$ & $2.82(1.53-5.19)$ & $.001^{*}$ & $1.05(0.67-1.65)$ & $>0.05$ \\
\hline
\end{tabular}

\footnotetext{
*: Statistically significant
}

DASS: Depression, Anxiety, and Stress Scales 
Table 4 showed logistic regression analysis for prediction of DASS subscale in HCWs and showed that the most significant predictive factors for depression were change in weekly vacation during COVID 19, followed by working in fever and chest hospitals. In addition to not receiving training regarding dealing with COVID 19 cases, history of death of one of their colleagues, history of antipsychotic drugs, and finally female gender.

The most significant factors for prediction of anxiety among the studied HCWs were change in weekly vacation during COVID 19, followed by taking antipsychotic medications, and working in fever hospitals. Other significant factors were history of death of colleagues, female gender, working in critical departments, age $<40$ years old, being a physician, and direct involvement in dealing with COVID 19 patients.

The most significant factors for prediction of stress among the studied HCWs was working as nurses, age $<40$ years, and single. Change in work schedules like weekly vacation during COVID 19 , changeable working shifts and working experience less than ten years are also significant independent factors.

\section{Discussion}

Health care workers play a crucial role during the pandemic that makes them more susceptible to anxiety and stress due to overwhelming health care systems (Temsah et al., 2020). The current work aimed to study the psychological impact of COVID-19 pandemic on health care workers of different specialties working in Egypt through using an online survey distributed through different online professional groups. There were varying degrees of psychological disturbances among study participants in the form of depression ranging (41.5\%), anxiety (67\%), and stress (27.8\%) ( Figure 1). A finding that is clearly related to the high work overload, long daily working hours, exposure to critical cases fighting for life, hard situations of taking critical decisions concerning patients' lives, the feeling at risk of getting infected and the fear of transmitting the disease to families. This finding coincides with that of Lai et al (2020) who reported that depression symptoms $(50.4 \%)$, stress $(71.5 \%$.) and anxiety $(44.6 \%)$ were present among health care 
workers working in Covid-19 hospitals of China. While the study done by Yeen and Zhao (2020) detected lower rates of anxiety and depression (35.6\% and $19.8 \%$ respectively), when compared to the current work.

Moreover, Zhang et al., (2020) studied the mental health and psychosocial problems of medical health workers in comparison to nonmedical health workers during the COVID-19 epidemic in China. They revealed a prevalence of insomnia (38.4 vs. $30.5 \%$, $\mathrm{p}<0.01)$. They also reported, anxiety (13.0 vs. $8.5 \%, \mathrm{p}<0.01$ ), depression (12.2 vs. $9.5 \% ; p<0.04)$, somatization (1.6 vs. $0.4 \%$; p $<0.01$ ), and obsessivecompulsive symptoms (5.3 vs. $2.2 \%$; $<0.01$ ).

The current work revealed statistically significant higher DASS and burnout scores among health care workers younger than 40 years (Table 2) that is attributed to the less practical experiences among younger HCWs compared to the older ones. Similar results were obtained by Yeen and Zhao (2020) during their web-based survey study concerning the generalized anxiety disorder, depressive symptoms and sleep quality during COVID-19 outbreak in China.
In addition, female HCWs experienced high DASS and burnout scores (Table 2). A finding that can be explained by the fact that gender has been shown to have a significant influence on burnout as well as somatic symptoms as the feminine hormones amplify the magnitude of stress responses. This is in concordance with the finding of EL Dalatony et al. (2019) who concluded that female nursing in Menoufia University hospitals expressed significant high stress score. Also, Verma et al. (2018) stated that female nursing had higher scores on most of the Standard Shift work index scales, in terms of disturbed sleep, fatigue, and poor psychological health. Social relations as being a member of a family play an important role in the emotional stability of health care workers. The study revealed higher levels of DASS and burnout for the single and divorced compared to the married women (Table 2). The work of Elbay et al. (2020) studied depression, anxiety and stress levels of physicians and its associated factors in Covid-19 pandemics in Turkey and detected that the DASS scale scores were higher among those who live alone compared to those living with their spouse and children. 
Meanwhile, the current study revealed that history of chronic diseases or taking antipsychotic drugs had significant impact on DASS scores (Table 2). This could be due to that chronic medical conditions subject the health care workers to a higher risk of acquiring severe and fatal COVID-19 infections. The study of Lai et al. (2020) and Zhu et al. (2020) stated that people with concomitant chronic noncommunicable diseases have higher risk of depression.

Further, the present work reported statistically significant higher DASS and burnout scores for health care workers with less than 10 years of work (Table 3). Being younger than 40 years with less than 10 years work duration may be associated with lack of experience and difficult decision making especially those concerning unexpected critical work situations. Therefore, we expected that younger healthcare workers could be easily subjected to psychological distress when facing new challenges. On the other hand, the study of Zhu et al. (2020) revealed that working for more than 10 years was associated with high level of stress, depression, and anxiety for female health care workers during the COVID-19 outbreak.
The current study showed that nurses experienced higher scores on DASS (Table 3). Nurses are especially vulnerable to job-related hazards and face a significant amount of emotional stress in relation to their jobs. This is even more important during a pandemic of an infectious disease ( Elbay et al. (2020) and Zhu et al. (2020)). Many other studies support this finding as that of Lai et al. (2020) who reported that Chinese health care workers exposed to COVID-19 especially female nurses have a high risk of developing unfavorable mental health outcomes.

Working in fever and chest hospitals was associated with significant high depression and burnout scores (Table 3). This is explained by the fact that fever and chest hospitals are the frontline health care facilities. As such, facilities faced the early rush of cases being a specialized tertiary care facility that is well prepared for diagnosis and management of suspected and confirmed COVID-19 cases especially at the beginning of the disease before enrollment of other governmental hospitals and private facilities. This is in agreement with Lai et al. (2020), which indicated that frontline nurses treating patients with COVID-19 are 
more likely to be exposed to the highest risk of infection because of their close, frequent contact with patients and working longer hours than usual. Working in critical departments (Table 2) and direct involvement in dealing with COVID-19 patients (Table 3) were associated with higher levels of depression, anxiety, stress, and burnout. HCWs in the emergency and ICU environment reported the highest rates of job burnout due to exposure to an excessive amount of work-related stress. Elshaer et al. (2018) reported that the contributing factors to high risk of burnout include intensive patient care, high mortality rates and inappropriate work conditions as high workload. Similarly, many other studies such as that of Kim and Choi (2016); Barello et al. (2020); Moccia et al. (2020); and Talaee et al. (2020) suggested that the probability of anxiety and depression of front-line medical staff in respiratory departments, emergency departments, intensive care units, and infection control departments is twice as high as that of nonclinical medical staff. Moreover, working more than 40 hours weekly was associated with high level of stress (Table 3) due to physical and psychological exhaustion. Additionally, significant association was detected between rotating work shift and high score on DASS scale for both depression and anxiety and for burnout score (Table 3). The study of Birhanu et al. (2018) confirmed our findings as it proved that health professionals working 50 hours and more per week and in night shift occasionally were more likely to develop workplace stress. Rotating work shift may lead to disruption of circadian rhythm and the sleep-wake cycle, job stress, as well as unsatisfactory work performance (Wisetborisut et al., 2014).

Receiving training regarding infection prevention and control of COVID-19 was associated with significant lower DASS and burnout scores (Table 4). This could be attributed to the better understanding of disease and infection prevention strategies that helps health care workers to feel safe and emotional stable. This is supported by Shaukat et al. (2020) study's results which proofed that the adequate provision and training on the use of personal protective equipment, strict infection control practices, shorter shift length, and provision of mental health and support services helps effectively in lowering the health consequences of dealing with COVID-19 patients. 
The most significant factors for prediction of stress, depression and anxiety among the studied HCWs were being a female, nurse with history of antipsychotic medication, with less than 40 years old, single, less than 10 years of experience, working in fever and chest hospitals with changeable working shifts system, changing vacation plans, in addition to inadequate training on dealing with COVID-19 pandemic and finally the presence of history colleague's death due to COVID-19 disease (Table 5). Those findings totally agree with those of Huang and Zhao (2020) who suggested that age and time spent focusing on COVID-19 may be potential risk factors for the psychological problems of the public. Younger participants $(<35$ years) were more likely to develop anxiety and depressive symptoms during COVID-19 outbreak than older participants $(\geq$ 35 years). Similarly, the results of multivariate analysis for prediction of depressive symptoms among health care workers done by (Wilson et al., 2020) revealed that female gender plays a role as a predictor of depressive symptoms. While female gender, fewer years of experience and hostel/temporary accommodation, act as predictors for anxiety symptoms through the results of multivariate analysis. Working hours less than 40 hours per week was associated with significant low scores for stress and anxiety (Table 5). This is in accordance with the study done by El Dalatony et al. (2019) who found that number of the working hours/week is one of independent significant factors that are associated with stress among the study group. These independent factors contributing to psychological disturbances among the study participants should be considered while designing a comprehensive mental health program targeting health care workers particularly frontline health care workers with direct exposure to suspected and confirmed COVID-19 disease with its subsequent physical and emotional stress.

\section{Conclusion}

Health care workers had varying degree of psychological stress related to work especially those in direct relations to healthcare of COVID-19 patients. Providing the HCWs with adequate support in terms of increasing their awareness, encouraging, and motivating them, and acknowledging their importance, and modifying working conditions especially in the era of COVID-19 


\section{Conflict of Interest}

None declared.

\section{Funding Sources}

\section{No Funding sources}

\section{Acknowledgement}

All Authors acknowledge and appreciate the considerable efforts of all health care workers as frontline fighters facing the COVID-19 pandemic and acknowledge the respectable participants in this current work.

\section{References}

1. Barello S, Palamenghi L and Graffigna G (2020): Burnout, and somatic symptoms among frontline healthcare professionals at the peak of the Italian COVID-19 pandemic. Psychiat Res; 290:113129. https://doi.org/10.1016/j. psychres.2020.113129.

2. Barry M, Al Amri M and Memish ZA (2020): COVID-19 in the Shadows of MERS-CoV in the Kingdom of Saudi Arabia. J Epidemiol Glob Health; 10(1):1-3. https://doi.org/10.2991/ jegh.k.200218.003

3. Birhanu M, Gebrekidan B, Tesefa G and Tareke M (2018): Workload Determines Workplace Stress among Health Professionals Working in Felege-Hiwot Referral Hospital, Bahir Dar, Northwest Ethiopia. J Environ Public Health.; Article ID 6286010 | 8 pages | https://doi. org/10.1155/2018/6286010

4. Blake H, Bermingham, F, Johnson G and Tabner A (2020): Mitigating the Psychological Impact of COVID-19 on Healthcare Workers: A Digital Learning Package. Int J Environ Res Public Health; 17(9):2997. https://doi.org/10.3390/ ijerph17092997
5. Centers for Disease Control and Prevention (CDC) (2013): Open Source Epidemiologic Statistics for Public Health. Version 3.01 Updated April 6, 2013. https://www.openepi.com/SampleSize/ SSPropor).

6. El Dalatony MM, Hathout HM and Allam HK (2019): Stress and Burnout among Nurses Working in Menoufia University Hospitals. Ergonomics Int J; 3(3):53-61. https:// doi:10.23880/eoij-1600020753

7. Elbay RY, Kurtulmuş A, Arpacıoğlu S and Karadere E (2020): Depression, anxiety, stress levels of physicians and associated factors in Covid-19 pandemics. Psychiat Res; 290:1131303. https:// doi.org/10.1016/j.psychres.2020.113130

8. Elshaer NS, Moustafa MS, Aiad MW and Ramadan MIE (2018): Job stress and burnout syndrome among critical care healthcare workers. Alexandria J Med; 54:273-7. https:// doi: 10.1016/j.ajme.2017.06.004

9. Fernandez PR, Lord H, Halcomb PE, Moxham PL, Middleton DR, et al. (2020): Implications for COVID-19: a systematic review of nurses' experiences of working in acute care hospital settings during a respiratory pandemic. Int J Nurs Stud; 111: 103637. https://doi. org/10.1016/j.ijnurstu.2020.103637.

10. Huang Y and Zhao N (2020): Generalized anxiety disorder, depressive symptoms, and sleep quality during COVID-19 outbreak in China: a web-based cross-sectional survey. Psychiat Res; 291, September 2020, 113190291.https:// doi.org/10.1016/j.psychres.2020.113190

11. Kang P, Lv Y, Hao L, Tang B, Liu Z, et al. (2015): Psychological consequences and quality of life among medical rescuers who responded to the 2010 Yushu earthquake: A neglected problem. Psychiat Res; 230(2): 517-23. https://doi. org/10.1016/j.psychres.2015.09.047

12. Kim JS and Choi JS (2016): Factors influencing emergency nurses' burnout during an outbreak of Middle East respiratory syndrome coronavirus 
in Korea. Asian Nursing Res; 10:295-9. https:// doi: 10.1016/j.anr.2016.10.002

13. Lai J, Ma S, Wang Y, Cai Z, Hu J, et al. (2020): Factors Associated with Mental Health Outcomes Among Health Care Workers Exposed to Coronavirus Disease 2019. JAMA Netw Open; 3(3): e203976. https://doi:10.1001/ jamanetworkopen.2020.3976

14. Li Z, Ge J, Yang M, Feng J, Qiao M, et al. (2020): Vicarious traumatization in the public, members, and non-members of medical teams aiding in COVID-19 control. Brain Behav Immun; 88:916-919 https://doi.org/10.1016/j. bbi.2020.03.007

15. Lovibond SH and Lovibond PF (1995): Manual for the Depression Anxiety Stress Scales. 2nd ed. Sydney: Psychology Foundation of Australia. Available at: https://www.scirp.org/reference/ ReferencesPapers.aspx? ReferenceID=1341416

16. Moccia L, Janiri D, Pepe M, Luigi D, Molinaro M, et al. (2020): Affective temperament, attachment style, and the psychological impact of the COVID-19 outbreak: an early report on the Italian general population. Brain Behav Immun (Elsevier); 87:75-9. https://doi. org/10.1016/j.bbi.2020.04.048.

17. Pappa S, Ntella V, Giannakas T, Giannakoulis VG, Papoutsi E, et al. (2020): Prevalence of depression, anxiety, and insomnia among healthcare workers during the COVID-19 pandemic: A systematic review and metaanalysis. Brain Behav Immun; 88:901-7. https:// doi.org/10.1016/j.bbi.2020.05.026

18. Preti E, Mattei V, Perego G, Ferrari F, Mazzetti $M$, et al. (2020): The Psychological Impact of Epidemic and Pandemic Outbreaks on Healthcare Workers: Rapid Review of the Evidence. Curr Psychiatry Rep; 22(8):43. https://doi.org/10.1007/s11920-020-01166-z.

19. Sestili C, Scalingi S, Cianfanelli S, Mannocci A, Del Cimmuto A, et al. (2018): Reliability and Use of Copenhagen Burnout Inventory in Italian
Sample of University Professors. Int J Environ Res Public Health. (MDPI); 15(8):1708. https:// doi.org/10.3390/ijerph15081708

20. Shaukat N, Ali DM and Razzak J (2020):Physical and mental health impacts of COVID-19 on healthcare workers: a scoping review. Int $\mathrm{J}$ Emerg Med; 13:40-7. https://doi.org/10.1186/ s12245-020-00299-5

21. Talaee N, Varahram M, Jamaati H, Salimi A, Attarchi M, et al. (2020): Stress and burnout in health care workers during COVID-19 pandemic: validation of a questionnaire. $\mathrm{J}$ Public Health (Berl.): From Theory to Practice; published online June 6, 2020. https://doi. org/10.1007/s10389-020-01313-z .

22. Temsah MH, Al-Sohime F, Alamro N, Al-Eyadhy A, Al-Hasan K, et al. (2020): The psychological impact of COVID-19 pandemic on health care workers in a MERS-CoV endemic country. $\mathrm{J}$ Infect Public Health; 13(6), 877-82. https://doi. org/10.1016/j.jiph.2020.05.021

23. Verma A, Kishore J and Gusain SA (2018): Comparative Study of Shift Work Effects and Injuries among Nurses Working in Rotating Night and Day Shifts in a Tertiary Care Hospital of North India. Iran J Nurs Midwifery Res; 23(1):51-6. https://doi.org/10.4103/ijnmr. IJNMR_15_17

24. Wilson W, Raj JP, Ra O, Ghiya M, Nedungalaparambi NM, et al (2020): Prevalence and Predictors of Stress, anxiety, and Depression among Healthcare Workers Managing COVID-19 Pandemic in India: A Nationwide Observational Study. Indian J Psychol Med; 42(4): 353-8. https://doi. org/10.1177/0253717620933992.

25. Wisetborisut C, Angkurawaranon W, Jiraporncharoen $\mathrm{R}$, Uaphanthasath $\mathrm{P}$ and Wiwatanadate (2014): Shift work and burnout among health care workers. J Occup Med; 64(4):279-86. https://doi.org/10.1093/occmed/ kqu009 
26. World Health organization (WHO) (2018): Global Health Observatory data repository, Health Workforce Statistics team, Egypt Health report; Updated 2020. Available at: https://www.who. int/docs/default-source/gho-documents/worldhealth-statistic-reports/6-june-18108-worldhealth-statistics-2018.pdf

27. Yeen H and Zhao N (2020): Generalized anxiety disorder, depressive symptoms and sleep quality during COVID-19 outbreak in China: a webbased cross-sectional survey. Psychiat Res; 288: 112954. (ELSEVIER). https://doi:10.1016/j. psychres.2020.112954

28. Zhang W, Wang K, Yin L, Zhao W, Xue Q, et al. (2020): Mental Health and Psychosocial Problems of Medical Health Workers during the COVID-19 Epidemic in China. Psychother Psychosom; 89:242-50. https:// doi:10.1159/000507639

29. Zhu Z, Xu S, Wang H, Liu Z, Zhang D ,et al. (2020): COVID-19 in Wuhan: Immediate Psychological Impact on 5062 Health Workers. E Clin Med; 100443. https://doi.org/10.1016/j. eclinm.2020.100443 